\title{
TÉCNICA DE LARSON ORIGINAL MODIFICADA PARA LA RECONSTRUCCIÓN DEL LIGAMENTO COLATERAL LATERAL DE LA RODILLA, REPORTE DE UN CASO
}

\author{
Morales Viteri Mario Carlos ${ }^{1}$, Llerena Freire Luis Francisco ${ }^{2 *}$, Benavides Vargas Karen Estefanía ${ }^{3}$, Álvarez \\ Guerrero Daniel Alejandro ${ }^{3}$, Heredia Montenegro Cristina Elizabeth ${ }^{3}$, Freire Pazmiño Dennise Solange ${ }^{3}$.
}

DOI: $10.48018 /$ rmv.v31.i2.15

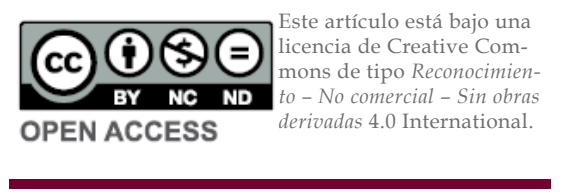

1. Hospital General IESS Ambato. Médico Tratante del Servicio de Traumatología y Ortopedia. Ambato-Ecuador 2. Hospital General IESS Ambato. Médico Residente del Servicio de Traumatología y Ortopedia. Ambato-Ecuador 3. Universidad Técnica de Ambato. Facultad de Ciencias de la Salud. Carrera de Medicina. Ambato - Ecuador.

\section{ORCID ID:}

Morales Viteri Mario Carlos

orcid.org/0000-0003-1984-8143

Llerena Freire Luis Francisco

orcid.org/0000-0002-8383-4099

Benavides Vargas Karen Estefanía orcid.org/0000-0001-5543-6686

Álvarez Guerrero Daniel Alejandro orcid.org/0000-0003-0301-099

Heredia Montenegro Cristina Elizabeth

orcid.org/0000-0003-0963-6294

Freire Pazmiño Dennise Solange

orcid.org/0000-0002-2807-2183.

*Corresponding author: Llerena Freire Luis Francisco

E-mail: pancho?ר_llerena@hotmail.com

Article history

Received: 20 - Jul - 2020

Accepted: 20 - Oct - 2020

Publish: 01 - Nov - 2020

CARE 2017 Check List statement: The author have real the CARE 2017 Check List and the manuscript was prepared and revised according to the CARE 2017 Checklist.

Conflict of interest: All authors declared that there are no conflicts of interest.

Financial disclosure: The authors have no financial relationships relevant to this article to disclose.

Authors' contribution: All the authors contributed in the search, selection of articles and writing. All the authors reviewed and approved the final manuscript.

Forma de citar este artículo: Morales Viteri MC, Llerena Freire LF, Benavides Vargas KE, Álvarez Guerrero DA,

Heredia Montenegro CE, Freire

Pazmiño DS. TÉCNICA DE LARSON ORIGINAL MODIFICADA PARA LA RECONSTRUCCIÓN DEL LIGAMENTO COLATERAL LATERAL DE LA RODILLA, REPORTE DE UN CASO. Rev Med

Vozandes. 2020; 31 (2): 107 - 113

\section{Resumen}

El ligamento colateral lateral (LCL), que forma parte de los estabilizadores de la rodilla, constituye una estructura importante dentro del complejo posterolateral de la misma (CPL), que restringe al varo, en los ángulos de flexión de esta, especialmente a los $30^{\circ}$, además de controlar la rotación externa. Son infrecuentes las lesiones que afectan a este complejo, etiológicamente entre el 50$90 \%$ se asocian a otras lesiones ligamentosas con predominio del $60 \%$ de daño del ligamento cruzado posterior. La técnica de Larson se basa en la reconstrucción del LCL y del complejo poplíteo, utilizando una inserción proximal única en el epicóndilo femoral.

Presentamos el caso de un paciente masculino de 28 años de edad, sin antecedentes clínicos, que acude debido a un varo forzado de la extremidad mientras realizaba actividad deportiva y tras recibir impacto directo en la región lateral interna de pantorrilla derecha, posterior a lo cual presenta dolor a los movimientos pasivos e imposibilidad a la deambulación. Además, presenta equimosis, edema, escoriaciones superficiales en cara lateral externa y dolor a la digitopresión en trayecto de ligamento colateral externo. A maniobras de exploración de rodilla: signo de bostezo en varo positivo, cepillado rotuliano positivo, hiperpresión de rotula positivo, limitación funcional a la flexión y extensión de la articulación.

Esta técnica, Larson modificada, resulta como una de las más prometedoras por sus varias características, como la menos exigente y al mismo tiempo brindando resultados alentadores. Este procedimiento se fundamenta en la reconstrucción del LCL y del tendón poplíteo (TP) en conjunto, resumiéndose así la reconstrucción del Ligamento Poplíteo Peroneo (LPP) como estabilizador estático del TP, con su inserción a nivel de la cabeza del peroné en conjunto con la del LCL y la restitución de ambas inserciones en el epicóndilo femoral, tomando en cuenta la morfología del peroné y los ángulos de movimientos de la rodilla.

Palabras clave: Complejo posterolateral, estabilizadores de la rodilla, ligamento colateral lateral, procedimiento quirúrgico, técnica de Larson. 
that affect this complex are infrequent, etiologically between $50-90 \%$ are associated with other ligamentous injuries with a predominance of $60 \%$ damage to the posterior cruciate ligament. The Larson technique is based on the reconstruction of the LCL and the popliteal complex, using a single proximal insertion in the femoral epicondyle.

We present the case of a 28-year-old male patient, with no medical history, who attended due to a forced varus of the $\operatorname{limb}$ while performing sports activity and after receiving a direct impact on the internal lateral region of the right calf, after which he presented pain to passive movements and inability to ambulation. In addition, he presents ecchymosis, edema, superficial abrasions on the external lateral aspect and pain on acupressure in the course of the external collateral ligament. A knee examination maneuvers: positive varus yawn sign, positive patellar brushing, positive patella hyperpressure, functional limitation to flexion and extension of the joint.

This modified Larson technique turns out to be one of the most promising due to its various characteristics, as the least demanding and at the same time providing encouraging results. This procedure is based on the reconstruction of the $L C L$ and the popliteal tendon (PT) as a whole, thus summarizing the reconstruction of the Peroneal Popliteal Ligament (LPP) as a static stabilizer of the PT, with its insertion at the level of the head of the fibula in conjunction with the of the $\mathrm{LCL}$ and the restitution of both attachments in the femoral epicondyle, taking into account the morphology of the fibula and the angles of movement of the knee.

Keywords: Posterolateral complex, knee stabilizers, lateral collateral ligament, surgical procedure, Larson's technique.

\section{Introducción}

El ligamento colateral lateral (LCL), constituye una estructura importante dentro del complejo posterolateral de la rodilla (CPL), y forma parte de los estabilizadores de la misma, restringiendo al varo, en todos los ángulos de flexión de la rodilla, especialmente a los $30^{\circ}$ y controlando la rotación externa. Se encuentra insertado proximalmente en el cóndilo femoral externo y distalmente en la región antero-lateral de la cabeza del peroné.'

Las lesiones que afectan el complejo postero-lateral son infrecuentes y entre el $50-90 \%$ se asocian a otras lesiones ligamentosas con una predominancia del $60 \%$ de afectación del ligamento cruzado posterior. La lesión del LCL es una de las menos frecuentes, se presenta en personas que realizan deportes de contacto y aquellos que requieren de cambios de dirección rápido. El mecanismo de lesión se produce con la pierna situada en rotación interna más una fuerza en varo. La evaluación de la integridad del $\mathrm{LCL}$ se realiza mediante la prueba de estrés en varo, la cual se efectúa en extensión completa de la rodilla y $30^{\circ}$ de flexión. Existen además exámenes de imágenes como la radiología simple, la dinámica y la resonancia magnética, que son importantes, para correlacionar los hallazgos con la clínica presente en el paciente..$^{2,3}$

Una vez que se realiza el diagnóstico, es de gran importancia evaluar el manejo que se debe aplicar según la gravedad de lesión del $L C L$, en el caso de ruptura completa de este ligamento la cirugía es necesaria, con el fin de evitar que se produzca una inestabilidad postero-lateral futura. Existen diversas técnicas de reparación y reconstrucción que pueden ser aplicadas; sin embargo, no existe un consenso sobre cuál de estas técnicas es la más adecuada. No obstante, muchos estudios reflejan que la reconstrucción muestra mejores resultados y menos tasas de fracaso en comparación con la técnica de reparación con resultados pocos favorables del 9 al $37 \% .{ }^{4}$

Entre las técnicas tenemos a la reconstrucción peronea y tibial de Laprade, tenodesis del bíceps, reconstrucción de Larson y técnicas con fascia lata o banda ilio-tibial entre otras. La técnica de Larson originalmente está basada en la reconstrucción del LCL y del complejo poplíteo, utilizando una inserción proximal única en el epicóndilo femoral. Esta técnica al tener presente el concepto de isometría de la cabeza del peroné con respecto al epicóndilo femoral en 
los ángulos de flexión, posee ventajas con respecto a otras técnicas en las que agregan reconstrucción a nivel de la parte posterior de la tibia. La modificación se basa en que se reproducen inserciones tanto del LCL como del Tendón Poplíteo (TP) a nivel del epicóndilo femoral y sus inserciones a nivel de la cabeza del peroné, de esta manera controla la inestabilidad postero-lateral y se restringe la inestabilidad en varo y rotación externa, presenta menor tasa de morbilidad, menor tiempo quirúrgico y mejores resultados postoperatorios..$^{5,6,7}$

El presente reporte de caso clínico tiene como finalidad demostrar la efectividad de la técnica quirúrgica de Larson como una de las más prometedoras por sus varios beneficios en el tratamiento de las lesiones del ligamento colateral lateral de la rodilla.

El estudio sigue las recomendaciones CARE, 8 para el reporte de casos clínicos, además, el paciente firmo el consentimiento informado autorizando su publicación.

\section{Presentación de caso}

Paciente masculino de 28 años de edad, sin antecedentes clínicos y quirúrgicos de importancia. Acudió al servicio de emergencia por presentar un cuadro de dolor, limitación funcional e inestabilidad lateral en la rodilla derecha de 2 días de evolución, producto de un impacto directo en la región lateral interna de pantorrilla que provocó un varo forzado de la extremidad. A través del examen físico se evidenció equimosis, edema, excoriaciones superficiales en cara lateral externa y dolor a la digito presión en trayecto de ligamento colateral externo; En las maniobras de exploración de rodilla se encontró el signo de bostezo externo, cepillado e hiperpresión de rótula positivos, además, limitación funcional a la flexión y extensión de la articulación.

El examen de laboratorio determinó un recuento leucocitario de $9.40 \mathrm{M} / \mathrm{uL}$, hemoglobina de $17.2 \mathrm{~g} /$ $\mathrm{dl}$, hematocrito $47.4 \%$, recuento plaquetario $343 \mathrm{k} /$ UL, neutrófilos 66.2\%, TP: 10.6, TTP: 21.4, glucosa 87,2 $\mathrm{mg} / \mathrm{dL}$, creatinina: $0,9 \mathrm{mg} / \mathrm{dL}$, VDRL y VIH negativos.

En la radiografía anteroposterior y lateral de rodilla derecha no se evidenció una lesión ósea, por tal motivo se realizó una resonancia magnética de rodilla para reforzar la interpretación diagnóstica, teniendo como resultado la solución de continuidad completa del ligamento colateral lateral con signos inflamatorios a su alrededor. (Figura 1A, 1B y 1C)

El diagnóstico definitivo fue una ruptura completa del ligamento colateral externo de la rodilla derecha, instaurando como tratamiento inicial analgesia (Paracetamol 1 gramo vía intravenosa cada 8 horas, Ketorolaco 30 miligramos vía intravenosa por razones necesarias), inmovilización con férula de rodilla y método RICE (reposo, hielo, compresión y elevación) de la extremidad afectada; Después de obtener una disminución importante del complejo secundario se ejecutó la resolución quirúrgica al tercer día.

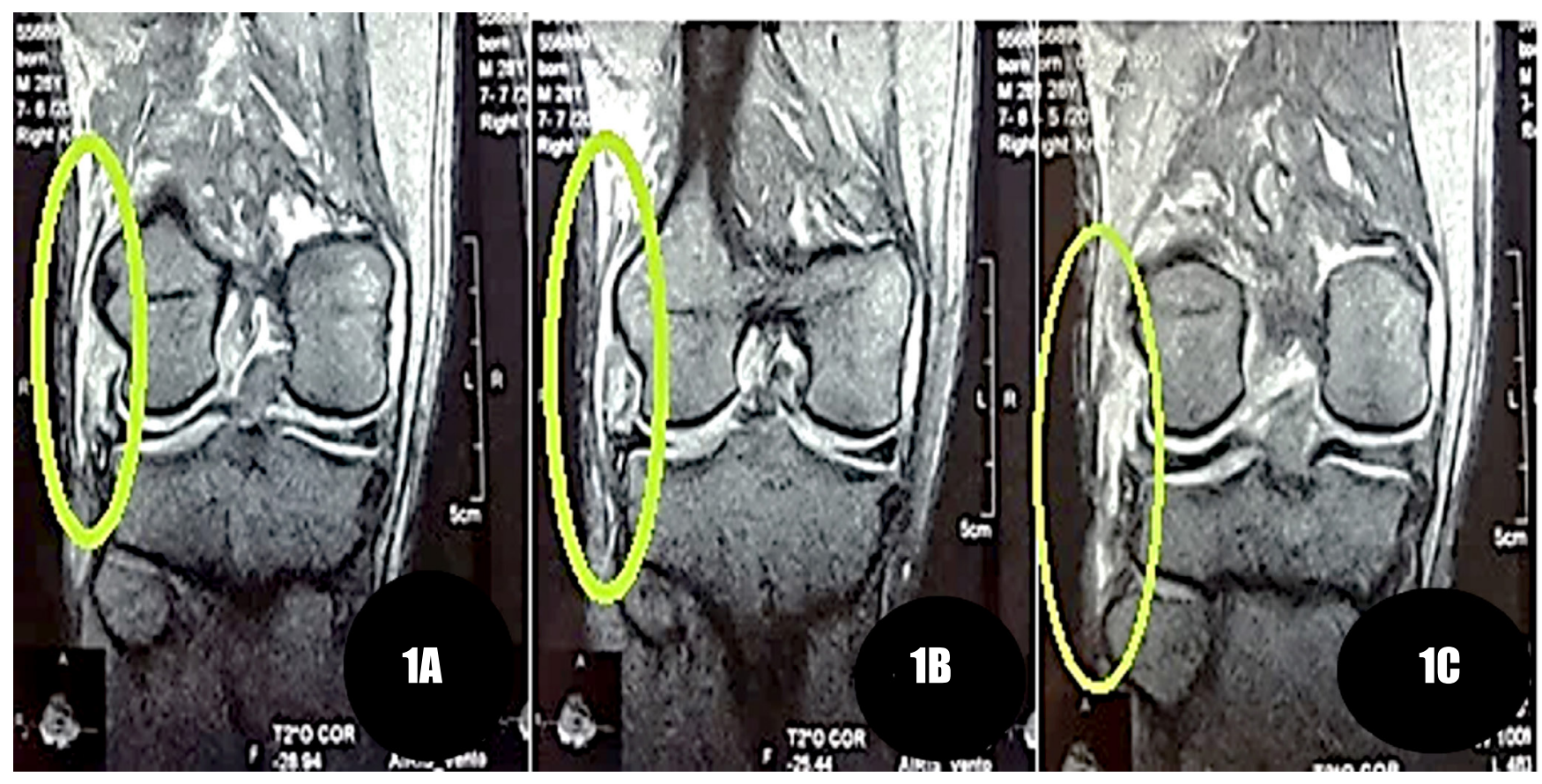

Figura 1A, 1B, 1C. Método diagnóstico de Lesión de Ligamento colateral lateral. RM que muestra solución de continuidad y signos inflamatorios alrededor del LCL de rodilla derecha. 


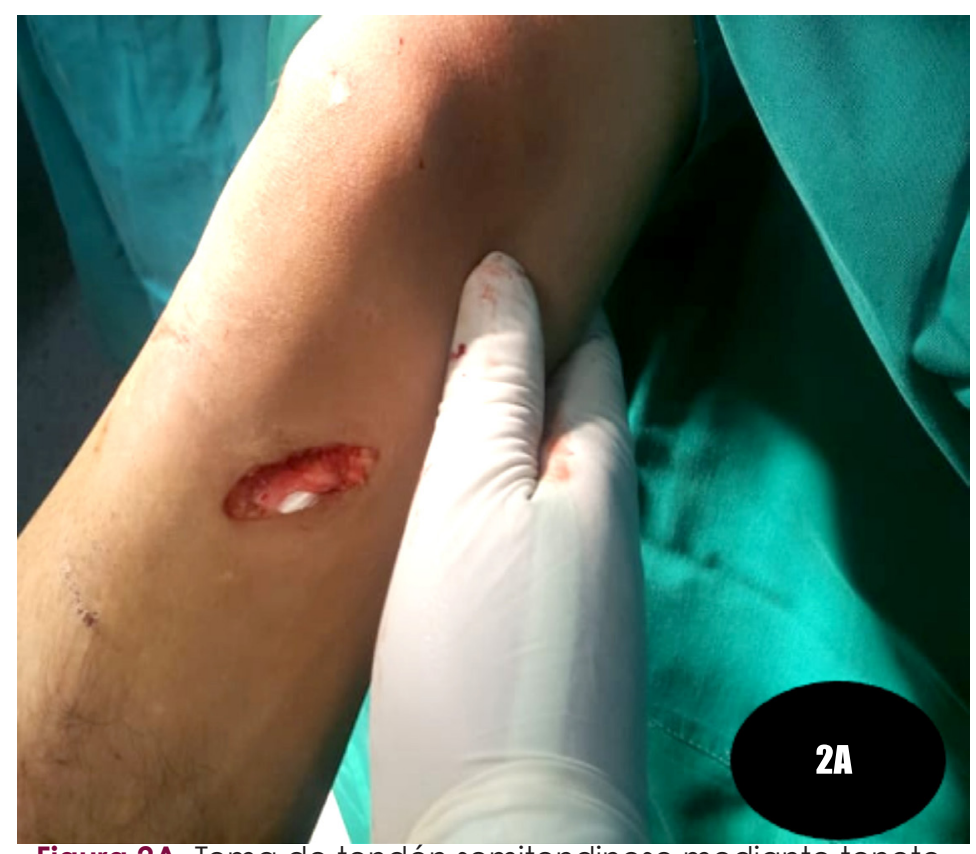

Figura 2A. Toma de tendón semitendinoso mediante tenotomo y desinsertándolo de su inserción distal.

Fuente: Elaborado por los autores

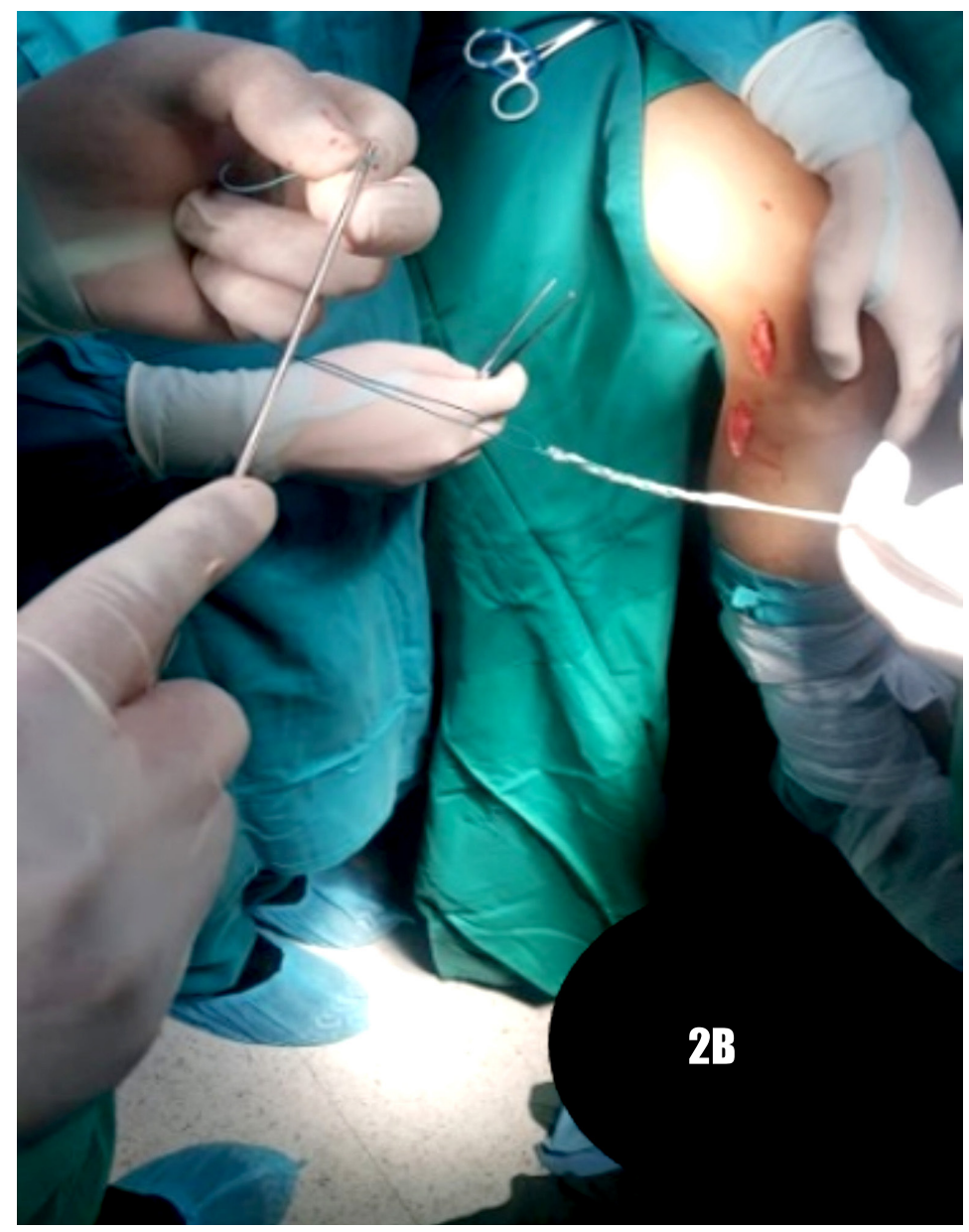

Figura 2B. Injerto preparado con puntos tipo krackow en cada extremo libre.

Fuente: Elaborado por los autores
Diagnóstico prequirúrgico

Ruptura completa del ligamento colateral externo de la rodilla derecha.

\section{Diagnóstico posquirúrgico}

Ruptura completa del ligamento colateral externo de la rodilla derecha.

\section{Diagnóstico posquirúrgico}

Ruptura completa del ligamento colateral externo de la rodilla derecha.

\section{Cirugía programada}

Reconstrucción del ligamento colateral externo de la rodilla derecha.

\section{Cirugía realizada}

Reconstrucción del ligamento colateral externo de la rodilla derecha con la técnica de Larson original modificada.

\section{Técnica quirúrgica}

Con el paciente en decúbito dorsal y la colocación de torniquete hemostático a nivel de región proximal del muslo derecho, se realizó cirugía mediante valoración artroscópica utilizando portales anteriores para la resolución de lesiones intra- articulares, así como también para evaluar la estabilidad del compartimiento externo. Posterior a la etapa artroscópica se procedió a la toma de tendón semitendinoso mediante tenotomo y desinsertándolo de su inserción distal, se obtuvo un injerto de entre 18 y $22 \mathrm{~cm}$ de largo, el cual fue preparado con puntos tipo krackow en cada extremo libre. (Figura 2A y 2B)

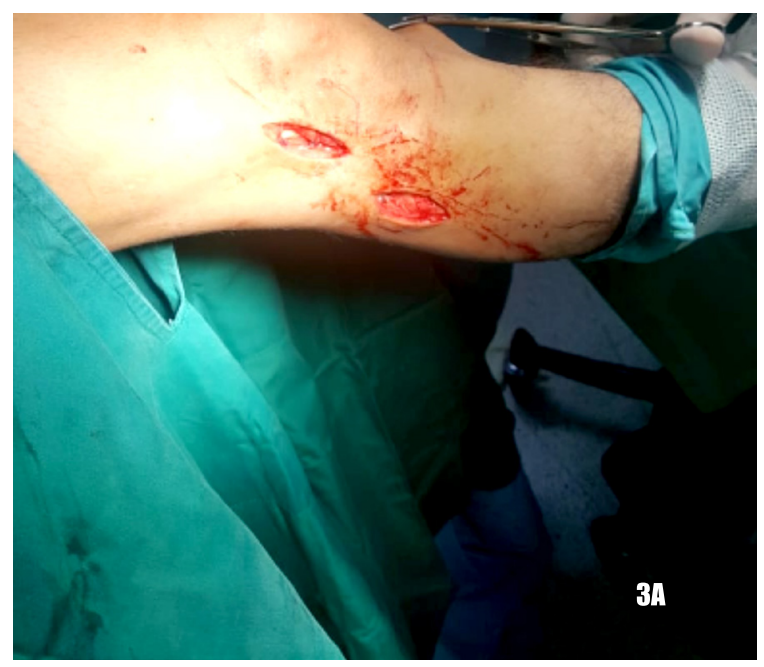

Figura 3. Abordaje quirúrgico.

Fuente: Elaborado por los autores

Se realizaron 2 incisiones lineales en cara lateral de rodilla derecha, una a nivel de cabeza de 

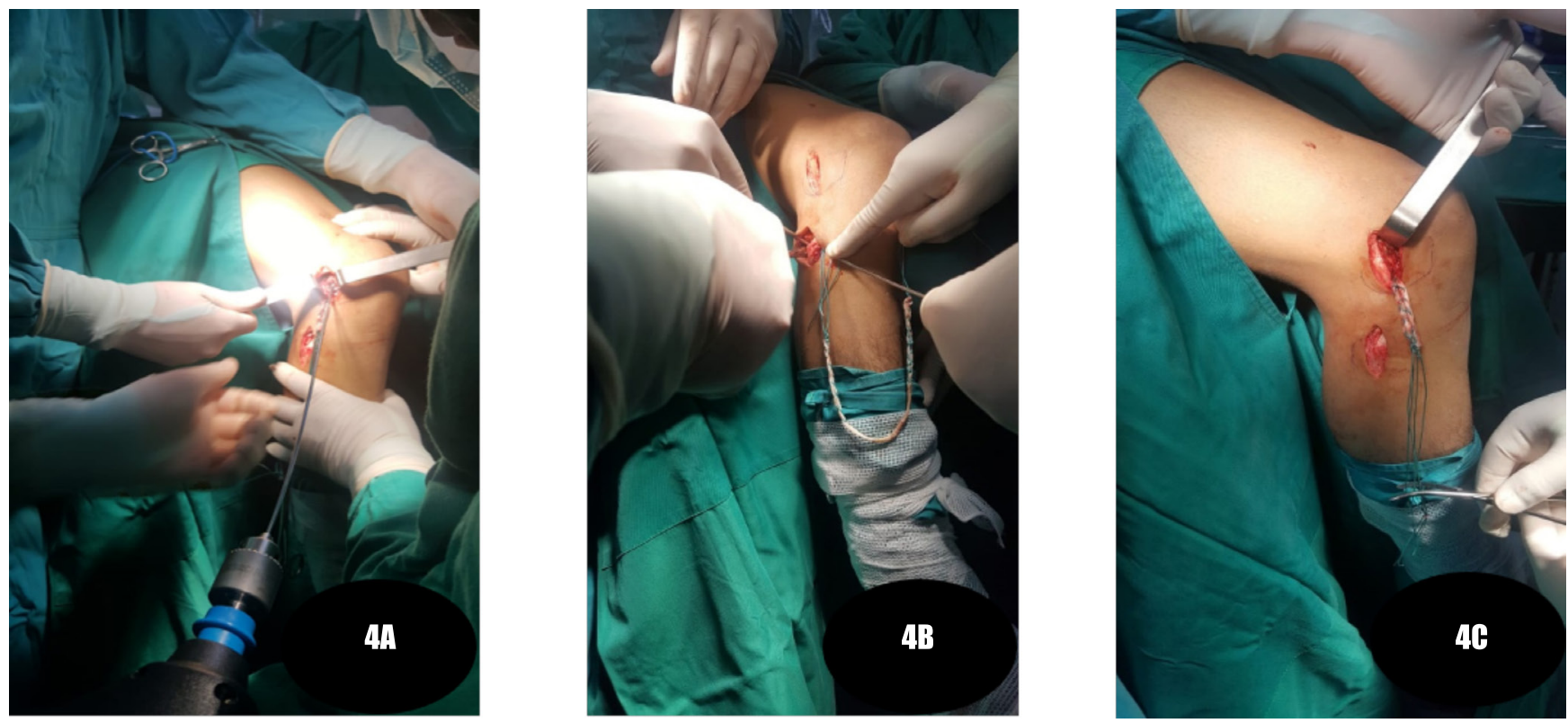

Figura 4A. Tunelización de la cabeza del peroné. B-C. Pase de injerto por túnel realizado en peroné.

peroné y otra a nivel de epicóndilo femoral. Disecamos el tejido celular subcutáneo hasta completar la incisión, lvego se reconoció y reparo el ciático poplíteo externo, debido que se trabajó sobre la cabeza del peroné. (Figura 3)

Posteriormente se disecó el tejido cuidadosamente por planos exponiendo la cabeza del peroné y el epicóndilo femoral, protegiendo el ciático poplíteo externo se procedió a la tunelización del peroné utilizando una guía con ojal de reconstrucción de Ligamento cruzado anterior fija, ubicándola en la parte media de la cabeza del peroné, pasamos la guía de anterior a posterior con una inclinación de 25 grados de abajo hacia arriba. Después de comprobar que estamos en el centro de la cabeza se realizó la tunelización del peroné con una broca quirúrgica de 4.5 milímetros teniendo cuidado de su salida en la parte posterior del peroné. Mediante un pasa tendón se pasó el injerto a través del túnel realizado. (Figura 4A, 4B y 4C)

Se identificó la inserción del LCL en el epicóndilo femoral, a ese nivel pasamos la guía con una inclinación de 45 grados a través de los cóndilos femorales, después de comprobar la adecuada posición de la guía se realizó la tunelización del fémur con una broca quirúrgica de 8 milímetros, garantizando siempre que el agujero en la cortical femoral lateral al ser de diámetro pequeño, deje una zona de seguridad que permita usar una fijación cortical. Utilizando el pasa tendón realizamos el mismo procedimiento que en el peroné. Para traccionar el injerto se utilizó las suturas de alta resistencia (dos) y para ajustar se realizó un nudo quirúrgico con las suturas blancas (dos), con lo que se comprobó de forma directa el paso del injerto a través del túnel femoral completo. Se evaluó la isometría mediante movimientos de flexo-extensión, seguros de haber logrado la isometría buscada se fijo el injerto en el fémur con un tornillo de interferencia bioabsorbible de 9 milímetros de diámetro y 30 milímetros de largo. (Figura 5A,5B y 6)

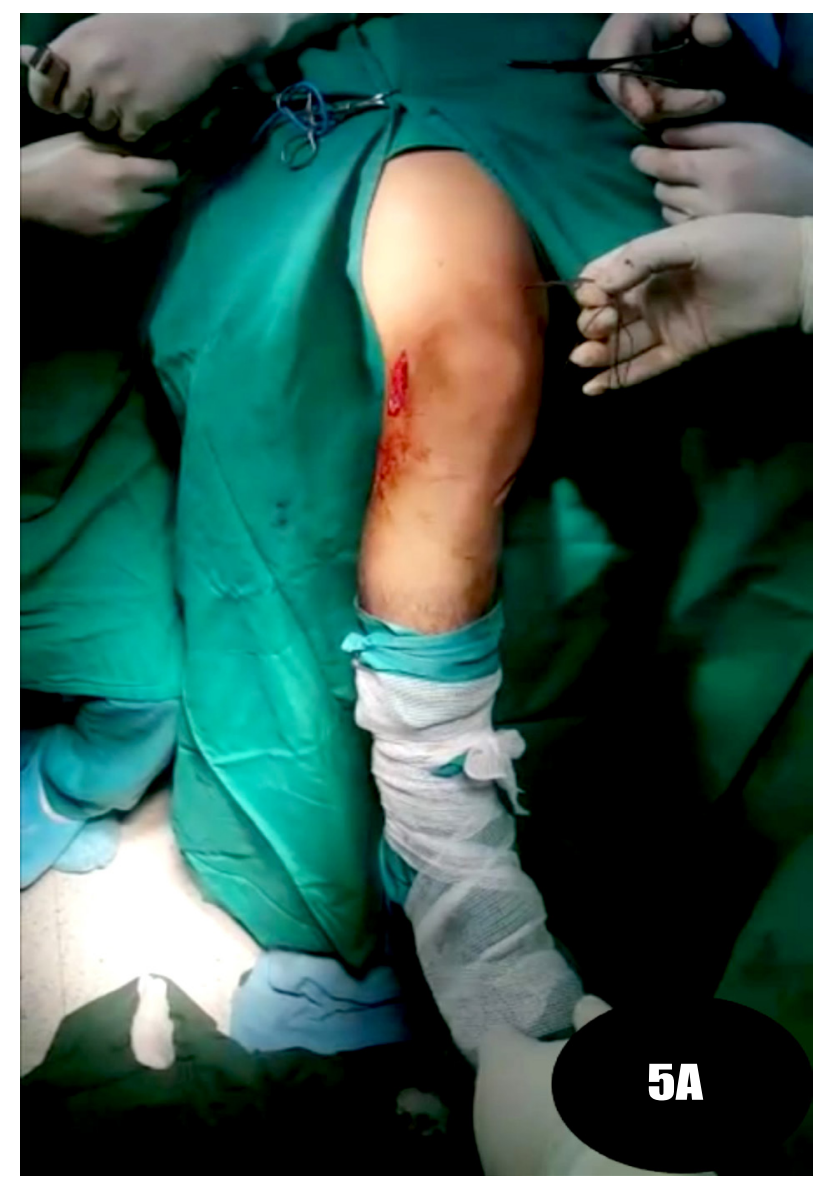

Figura 5A. Tracción de injerto para temple y fijación con suturas de alta resistencia en cortical femoral interna. B: Colocación de Tornillo de interferencia biodegradable.

Fuente: Elaborado por los autores 


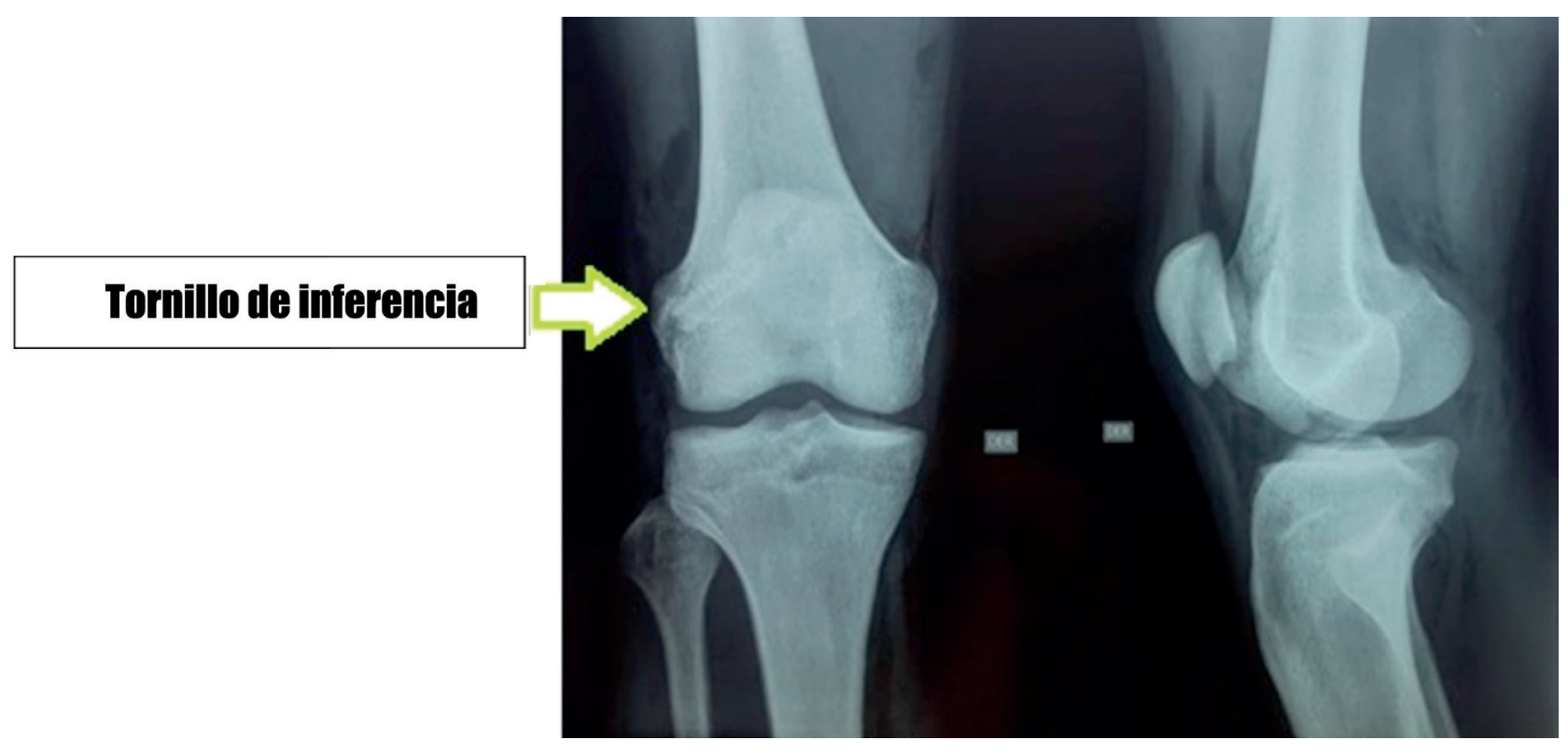

Figura 6. Control radiográfico.

En el manejo posquirúrgico que se realizó en este paciente fue antibioticoterapia profiláctica quirúrgica (Cefazolina 1 gramo vía intravenosa cada 8 horas por 3 días), analgesia (Paracetamol 1 gramo vía intravenosa cada 8 horas por 5 días, Ketorolaco 30 miligramos vía intravenosa cada 8 horas por 2 días) e hidratación (Lactato Ringer 1000 mililitros vía intravenosa 60 $\mathrm{ml} / \mathrm{h}$ por 3 días). Al cuarto día sin evidencia de complicaciones ulteriores el paciente fue dado de alta; se indicó la utilización de inmovilizador de rodilla por 4 semanas con apoyo parcial y posterior a este tiempo inicio de fisioterapia, que tiene como objetivo permitir la flexión de 90 grados de la articulación de la rodilla, la misma que se realizó por parte del departamento de fisiatría.

\section{Discusión}

Existen estructuras anatómicas estáticas y dinámicas que en conjunto conforman el denominado complejo posterolateral de la rodilla, este se encarga de proporcionar estabilidad en el eje postero-lateral de la misma y evita la inestabilidad que puede verse en varo y rotación externa. Las estructuras que destacan son el ligamento lateral externo o también llamado colateral lateral, el tendón poplíteo y el ligamento poplíteo fibular.

El Ligamento colateral lateral puede ser identificada como una estructura anatómica estática cuya función es estabilizar el varo de la rodilla. La inserción proximal se da en un punto en la región posterior y proximal del epicóndilo femoral y la distal a nivel de la región anterior lateral de la cabeza del peroné.?

El presente artículo muestra el caso de un paciente con diagnóstico de ruptura de ligamento colateral lateral, el daño de esta estructura causa inestabilidad y desgaste a nivel de la articulación con la posterior afectación de la funcionalidad en los pacientes, provocando un alto grado de discapacidad para el desarrollo normal de sus actividades, el LCL es el primer restrictor a la desviación del eje en varo, en especial a $30^{\circ}$, a la rotación externa y a los mismos grados en flexión. Aproximadamente del 50 al 90 \% de esta lesión se relaciona con daño de otros ligamentos de la rodilla, especialmente del ligamento cruzado anterior y del ligamento cruzado posterior, en varias ocasiones la falla en la reparación de estos ligamentos es la omisión de la ruptura del LCP. ${ }^{4}$

El diagnóstico de la afectación ligamentosa de la rodilla se lo realiza mediante un examen físico exhaustivo en donde se apliquen maniobras de hiperextensión, rotación externa y varo forzado, estas maniobras se clasifican en estáticas y dinámicas de acuerdo a la estructura anatómica afectada, cuando existe una ruptura del ligamento colateral lateral la técnica utilizada es de tipo dinámica, la denominada bostezo en varo, la misma que fue aplicada por el personal médico a cargo del caso presentado en el artículo, además de maniobras como la de cepillado rotuliano, hiperpresión de rotula, limitación funcional a la flexión y extensión de la articulación que también fueron positivas. ${ }^{4}$

Es necesario que además de la sospecha clínica y el examen físico se utilice técnicas de imagen como la radiografía, que es importante para la identificación de este tipo de lesiones, con esta técnica de imagen se puede visualizar el llamado signo del arcuato un arrancamiento de la apófisis estiloides del peroné. La detección temprana permite planificar una cirugía y mejora el pronóstico del paciente. Existe además otros 
exámenes de imagen como la resonancia magnética que cumple un papel importante al identificar estructuras de significancia para el diagnóstico, además que ayuda en la diferenciación de una lesión crónica y una lesión aguda. Este tipo de examen tiene una sensibilidad muy alta para reconocer la morfología de la patología. Es de gran relevancia relacionar los hallazgos tanto del examen físico y exploración clínica, como lo que se observa en las pruebas de imagen, como en la radiografía y en la resonancia magnética. ${ }^{10}$

Se han descrito varias técnicas reconstructivas, dentro del manejo quirúrgico de las lesiones del complejo posterolateral, cada una con resultados clínicos promisorios; sin embargo, se han encontrado problemas con la laxitud que se presenta en el posquirúrgico tardío. En la actualidad, el tratamiento se basa en diferentes variables que el cirujano debe tomar en cuenta al momento de planificar el resultado final posquirúrgico entre ellos: la estabilidad, laxitud y durabilidad del injerto. ${ }^{11,12}$

Una de las técnicas más promisorias, es la técnica de Larson modificada, debido a sus múltiples características, resultando una técnica menos exigente y a su vez brindando resultados favorables para el paciente. ${ }^{4}$ La técnica de Larson original se fundamenta en la reconstrucción del LCL y del complejo poplíteo en conjunto, dándose así la reconstrucción del Ligamento Poplíteo Peroneo (LPP) como sistema estático del TP, la restitución de las dos inserciones en el epicóndilo femoral, así como su inserción a nivel de la cabeza del peroné en conjunto con la del LCL, tomando en cuenta la morfología del peroné y los ángulos de movimientos de la rodilla..$^{6,13}$

La actualidad se emplea la técnica de Larson modificada difiere de la original en la que se utiliza una única inserción proximal a nivel del epicóndilo femoral, en la técnica las inserciones femorales se realizan a la altura del LCL y del TP mediante la reconstrucción del LPP con su sistema estático, a nivel de la cabeza del peroné, controlando de esta manera la inestabilidad postero-lateral y evitando a la vez la inestabilidad en varo y la rotación externa, producida por la lesión del complejo postero-lateral, disminuyendo la morbilidad al no tener que agregar la tunelización a nivel de la tibia, con un tiempo quirúrgico reducido y obteniendo con una técnica menos invasiva y más simple los mismos resultados y con la probabilidad de lograr un mejor resultado en cuanto a la biomecánica de la rodilla, respetando la isometría natural de estas estructuras anatómicas.5,14

Dentro de las técnicas quirúrgicas que toman en cuenta el concepto de la isometría anatómica de la cabeza del peroné en todos los ángulos de flexión de la rodilla con respecto a las otras estructuras anatómicas como el epicóndilo femoral y no con la parte posterior de la tibia proximal tenemos a la técnica de Larson modificada. Mientras que en otras técnicas quirúrgicas se reconstruye la porción dinámica del TP mediante un estabilizador estático. ${ }^{15,16}$

Por lo antes mencionado, se concluye que la reconstrucción con la técnica de Larson modificada tiene algunas ventajas con respecto a otras técnicas quirúrgicas, utilizadas para la reconstrucción del complejo postero-lateral a nivel de la tibia en la porción dinámica del TP, que reconstruyen con el componente estático; debido que mantiene la isometría anatómica normal, corrige el defecto de varo y rotación externa y permite un resultado favorable en los pacientes tratados a través de esta técnica.

\section{Referencias}

1. Chanla J, Moatshe G, Dean C, LaPrade R. Posterolateral Corner of the Knee: Current Concepts. Arch Bone Jt Surg. 2016 Abril; 4(2): p. 97-103.

2. Fitzpatrick D, Dursun M, Webborn N. Two Cases of Isolated Lateral Collateral Ligament Knee Strain in Footballers Presenting with Novel Mechanism of Injury and Novel Examination Findings. Arch Physiother Rehabil. 2019; 2(2): p. 41-45.

3. Shon OJ, Park J, Kim B. Current Concepts of Posterolateral Corner Injuries of the Knee. Knee Surg Relat Res. 2017 Diciembre 1; 29(4): p. 256-268.

4. Guerrero J, Cruz A. Conclusiones de la jornada de actualización sobre el abordaje en las lesiones del complejo posterolateral de la rodilla. Revista Española de Artroscopia y Cirugía Articular. 2018 Mayo; 25(62): p. 76-83.

5. Stannard J, Stannard J, Cook J. Repair or Reconstruction in Acute Posterolateral Instability of the Knee: Decision Making and Surgica Technique Introduction. J Knee Surg. 2015; 28 p. 450-454.

6. Franciozi $C$, Kubota $M$, Abdalla $R$, Cohen $M$ Malheiros M. Posterolateral corner repair and reconstruction: overview of current techniques. Annals of Joint. 2018; 3(89): p. 1-9.
Gómez Palomo JM. Lesiones del Ángulo Posterolateral de la Rodilla. Artículo de revisión. Rev. S. And. Traum. y Ort. 2019 Mayo; 36(1/4) p. 19-27.

8. Riley DS, Barber MS, Kienle GS, Aronson JK, von Schoen-Angerer T, Tugwell P, et al. CARE guidelines for case reports: explanation and elaboration document. J Clin Epidemiol. 2017. p: S0895-4356 (17) 30037-9.

9. Gigante F, Lapera M, Crifasi N, Trevisan G. Técnica de Larson Modificada Para la Reconstrucción del. Artroscopia. 2015; 20(4): p. 130-134.

10. Fernandez E, Hernandez G, Calatayud J, Exposito D, Guerra F. Esquina Posterolateral de la Rodilla : Aproximación Clínico -. Seram. 2019.

11. Afanador Acuna EW, Sánchez Villa FJ, Sánchez Cruz , Ibánez Pinilla. Resultados funcionales de pacientes con reconstrucción crónica de lesiones aisladas y combinadas del ligamento cruzado posterior. Elsevier Espana. 2016 julio; $30(2)$ : p. 67-76.

12. Drenck TC, Preiss, Domnick, Herbort, Frings. El bypass de Popliteus proporciona propiedades biomecánicas superiores en comparación con la técnica de Larson en la reconstrucción de la esquina combinada posterolateral y la lesión del ligamento cruzado posterior. SpringerLink. 2020 abril.

13. Feeley BT, Muller MS, Sherman, Allen, Pearle AD. Comparison of Posterolateral Corne Reconstructions Using Computer-Assisted Navigation. Arthroscopy: The Journal of Arthroscopic and Related Surgery. 2010 Agosto: $26(8)$.

14. Chahla J, Murray LR, Robinson J, Lagae K Margheritini F, Fritsc B, et al. Posterolateral cor ner of the knee: an expert consensus statement on diagnosis, classification, treatment and rehabilitation. PubMed. 2019 agosto 2520-2529

15. Dold AP, Swensen, Strauss, Alaia. The Pos teromedial Corner of the Knee: Anatomy Pathology, and Management Strategies Journal of the American Academy of Ortho paedic Surgeons. 2017; 25(11): p. 752-761.

16. Gelber PE, Erquicia JI, Abat F, Sosa G, Pelfort $X$, Tey $M$, et al. Técnicas de reconstrucción del ángulo posterolateral de la rodilla según el grado de lesión. Orientación de los túneles según un modelo cadavérico. Cuadernos de Artroscopia. 2012 Octubre; 19(47): p. 34-40. 